Supporting Information (AC0495891)

\title{
Fullerene-Functionalized Gold Nanoparticles: Electrochemical and Spectroscopic Properties
}

Fengjun Deng, ${ }^{\dagger}$ Yiyun Yang, ${ }^{\dagger}$ Sungho Hwang, ${ }^{\dagger}$ Young-Seok Shon ${ }^{7^{*}}$ and Shaowei Chen ${ }^{\dagger *}$

$\dagger$ Department of Chemistry, Southern Illinois University, Carbondale, Illinois 62901

$\$$ Department of Chemistry, Western Kentucky University, Bowling Green, KY 42101

The composition of particles was studied using FTIR spectroscopy and TGA. Infrared spectroscopy is a useful method for obtaining structural and conformational information regarding monolayers on metal particles. The presence of $\mathrm{C}=\mathrm{C}$ stretching band around at 1600 $\mathrm{cm}^{-1}$ is an indication of the presence of aromatic moiety. Figure S1 clearly shows multiple bands near $1600 \mathrm{~cm}^{-1}$, which indicates the presence of different $\mathrm{C}=\mathrm{C}$ functional groups in $\mathrm{C}_{60}$ and 4aminothiophenolate ligands of C60-C6AuATP.

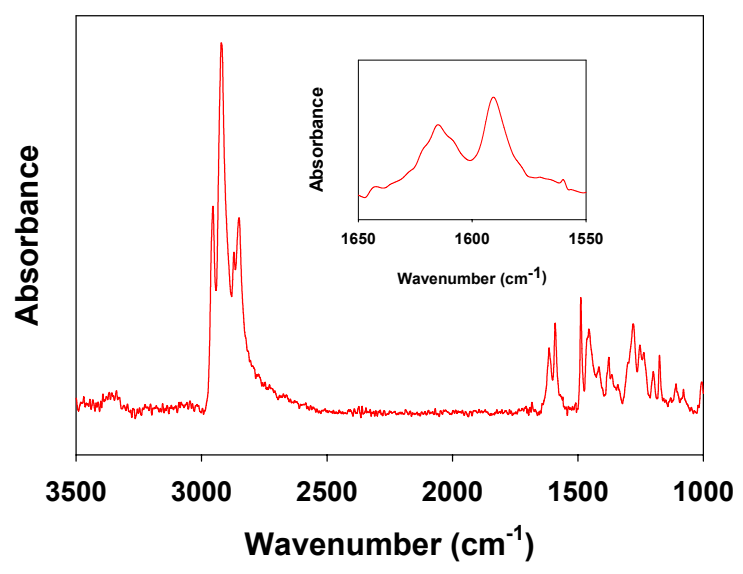

Figure S1

\footnotetext{
*To whom all correspondence should be addressed. E-mail: schen@chem.siu.edu
} 
It has been shown that TGA yields an organic weight fraction for the clusters. ${ }^{11}$ Since the amination reaction of $\mathrm{C}_{60}$ with $\mathrm{C} 6 \mathrm{AuATP}$ does not involve a loss of any molecule, the reaction should result in an increased organic weight fraction for C60-C6AuATP compared to C6AuATP. As shown in Figure S2, the increased fraction of $4.8 \%$ is corresponding to the added weight of tethered $\mathrm{C}_{60}$, which is estimated of $c a .6$ per Au particle.

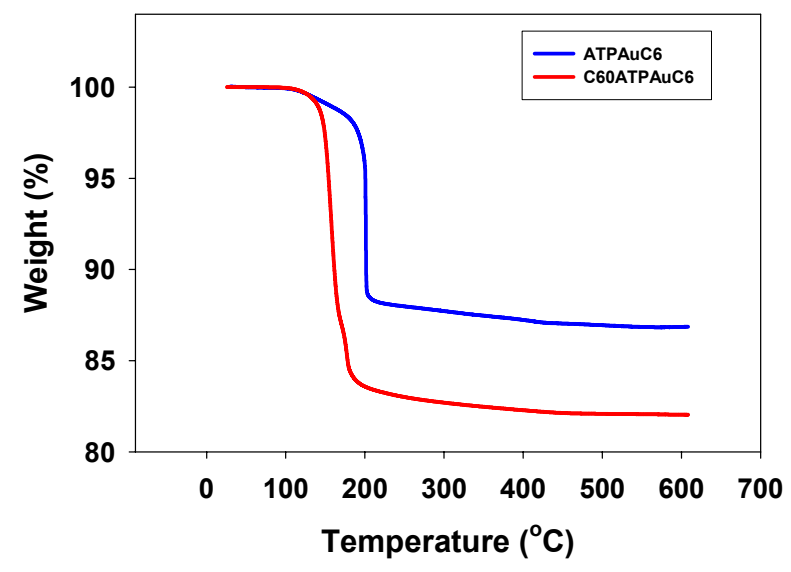

Figure S2 